\title{
14. Ueber den Druck der Lichtstrahlen ${ }^{1}$ ); von D. A. Goldhammer.
}

Bartoli ${ }^{2}$ ) hat auf Grund von thermodynamischen Betrachtungen das Resultat abgeleitet, dass die auf eine vollkommen reflectirende Fläche normal auffallenden Lichtstrahlen auf dieselbe einen Druck $p$ ausüben, welcher gleich ist der doppelten Energie $e$ in der Volumeneinheit der einfallenden Strahlen: $p=2 e$.

Für einen allseitig mit gleich temperirten, für Wärme undurchlässigen Wänden umgebenen, absolut leeren Raum (Vacuum) berechnete Boltzmann ${ }^{3}$ ), immer auf dem thermodynamischen Wege, auf eine absolut spiegelnde Fläche

$$
p=\frac{1}{3} e,
$$

indem er das Stefan'sche Strahlungsgesetz in Betracht zog. In einer späteren Arbeit über denselben Gegenstand bemerkt Boltzmann ${ }^{4}$, dass aus der Beziehung

$$
p=\frac{1}{3} e
$$

$p=e$ bez. $p=2 e$ folgen würde für normale Incidenz auf eine absorbirende bez. vollkommen reflectirende Fläche und dass dieses Resultat mit den Folgerungen aus der elektromagnetischen Lichttheorie Maxwell's übereinstimmt.

In der That lesen wir bei Maxwell den folgenden für nicht leitende Körper geltenden $\left.S_{a t z}{ }^{5}\right)$, ,in a medium in which waves are propagated there is a pressure in direction normal to the waves and numerically equal to the energy in unit of volum"; einige Zeilen weiter (Art. 793) äussert sich Maxwell in einer noch viel allgemeineren Weise, indem er sagt: , a flat

1) Aus den "Archives Néerlandaises", 1900; für die Ann. d. Phys. zum Teil neu bearbeitet vom Verfasser.

2) A. Bartoli, Sopra i movementi prodotti dalla luce e dal calore. 56 pp. Firenze 1876.

3) L. Boltzmann, Wied. Ann. 22. p. 31. 1884.

4) 1. c. p. 293 .

5) J. C. Maxwell, Treatise on electricity and magnetism, Second ed. 11. p. 401. Art. 792. 1881. 
body exposed to sunlight would experience this pressure on its illuminated side only, and would therefore be repelled from the side on which the light falls".

Diese Behauptung Maxwell's interpretirt nun Boltzmann in folgender Weise: "Maxwell's Resultat gilt," sagt er ${ }^{1}$ ), ,wenn der Strahl senkrecht auf die gedrückte Fläche auffällt und von derselben absorbirt wird. Würde er nahe senkrecht auffallen und unter demselben Winkel reflectirt, so wäre der Druck der doppelte." Natürlich sind hier Absorption und Reflexion als volikommen gemeint.

Wir sehen also, dass es zwei vollkommen roneinander unabhängige Wege zur Ableitung dieses sogenannten „Lichtdruckes" giebt: der thermodynamische und der elektromagnetische.

Nun schreibt Boltzmann selbst seinen Rechnungen einen ,vielfach provisorischen Charakter" $\mathbf{z u}^{2}$ ); Bartoli hält die Einführung des Lichtdruckes nur als ,l'ipotesi più semplice“"3); auch nach Boltzmann ${ }^{4}$ ) könnte der Widerspruch mit dem zweiten Hauptsatze, auch durch eine andere Hypothese gehoben werden".

Was aber die elektromagnetische Lichttheorie Maxwell's anbetrifft, so ist ohne weiteres klar, dass der oben angeführte (aus Art. 792) Satz noch nicht erlaubt, direct über die an der Grenze zweier Medien wirkende ponderomotorische Kräfte zu schliessen, denn auf eine Grenzfläche gegen einen anderen Körper wirkt durchaus nicht dieser Druck direct, sondern ,die Resultirende derjenigen Kräfte, welche sich nach der aus der Elasticitätstheorie bekannten Regel aus den im Innern eines jeden Körpers vorhandenen Spannungen ergeben. ${ }^{5}$ )

Nur in den Grenzfällen einer normalen Incidenz auf eine vollkommen reflectirende bez. vollkommen schwarze Fläche kann diese Resultirende direct angegeben werden: bei einer vollkommenen Reflexion sind alle elektromagnetische Vectoren im Innern des reflectirenden Körpers Null; bei einer voll-

1) L. Boltzmann, l. c. p. $291-292$.

2) 1. c. p. 293 .

3) A. Bartoli, 1. c. p. 25.

4) L. Boltzmann, 1. c. p. 38 .

5) Vgl. F. Pockels, Grunert's Archiv 12. p. 60. 1894. 
kommenen Absorption könnte man sagen, es existiren in dem absorbirenden Körper keine elektromagnetische Vectoren als solche. Diese Vorstellung giebt aber zu Bedenken Anlass: vielmehr muss man in diesem Falle die beiden sich angrenzenden Körper als optisch identisch — an der Trennungsebene betrachten. ${ }^{\text {) }}$

Diese Folgerungen gelten aber nicht ohne gewisse Beschränkungen; sie sind nämlich nur dann richtig, wenn thatsächlich die ponderomotorische Wirkung des Lichtes einzig nur aus dem von Maxwell abgeleiteten Drucke $p$ besteht, d. h. die elektromagnetischen Spannungen derart sind, dass sie keine auf innere Volumenelemente des Körpers wirkende Kräfte ergeben. Und dies ist im allgemeinen gerade nicht der Fall, wie es schon $\mathrm{Hertz}^{2}$ ) bemerkt hatte. Dann aber bleibt auch die Frage über den Lichtdruck selbst in den Grenzfällen der absoluten Reflexion bez. Absorption ganz offen.

1. Betrachten wir den allgemeinen Fall eines polarisirbaren, leitenden und sich mit den Geschwindigkeitscomponenten $\alpha, \beta, \gamma$ bewegenden Mediums, so lauten die Gleichungen der elektromagnetischen Lichttheorie nach $\mathrm{Hertz}^{3}$ ) und v. Hel mholtz ${ }^{4}$, indem man für homogene isotrope Körper

$$
\begin{array}{lll}
\mathfrak{Z}=\mu L, & \mathfrak{M}=\mu M, & \mathfrak{N}=\mu N, \\
\mathfrak{X}=\varepsilon X, & \mathfrak{V}=\varepsilon Y, & \mathbb{Z}=\varepsilon Z
\end{array}
$$

1) Für einen sich 2. B. im Vacuum befindenden Körper ist das Reflexionsvermögen

$$
J=\frac{(1-N)^{2}+K^{2}}{(1+N)^{2}+K^{2}}
$$

wenn $N$ den Brechungs-, $K$ den Absorptionscoefficienten bedeuten. Für eine schwarze Fläche muss $J=0$ sein, woraus folgt die optische Identität $N=1, K=0$. Dann existirt aber kein einseitiger Druck an der Trennungsebene.

Bei dieser Gelegenheit sei noch folgendes bemerkt: wenn ein Körper in Berührung mit zwei verschiedenen Körpern schwarz erscheint, so muss er offenbar optisch inhomogen sein. Das stimmt mit der bekannten Thatsache überein, dass Russ u. dgl. schwarze Körper sehr stark porös sind. Am einfachsten wären dieselben als eine Reihe von Hohlkörpern anzusehen. Vgl. F. Kurlbaum, Wied. Ann. 67. p. 885. 1899.

2) H. Hertz, Wied. Ann. 41. p. 398. 1890.

3) l. c. p. 374 .

4) H. v. Helmholtz, Wied. Ann. 47. p. 13.1892 
setzt, $\mu, \varepsilon$ als Constante betrachtet und die Bezeichnungen von Hertz benutzt,

$$
\begin{aligned}
& A \mu\left\{\frac{\partial L}{\partial t}+\frac{\partial}{\partial y}(\beta L-\alpha M)-\frac{\partial}{\partial z}(\alpha N-\gamma L)\right. \\
& \left.+\alpha\left(\frac{\partial L}{\partial x}+\frac{\partial M}{\partial y}+\frac{\partial N}{\partial z}\right)\right\}=\frac{\partial Z}{\partial y}-\frac{\partial Y}{\partial z},
\end{aligned}
$$

analog für $M, N$, und

$$
\begin{aligned}
A \varepsilon\left\{\frac{\partial X}{\partial t}\right. & +\frac{\partial}{\partial y}(\beta X-\alpha X)-\frac{\partial}{\partial z}(\alpha Z-\gamma X) \\
& \left.+\alpha\left(\frac{\partial X}{\partial x}+\frac{\partial Y}{\partial y}+\frac{\partial Z}{\partial z}\right)\right\}=\frac{\partial M}{\partial z}-\frac{\partial N}{\partial y}-4 \pi A u,
\end{aligned}
$$

analog für $Y$ und $Z$.

2. Wir wollen zunächst die Werte der ponderomotorischen Kräfte im Innern unseres Mediums auf etwas anderem Wege ableiten, als es bei Hertz und v. Helmholtz der Fall war. $\mathrm{Zu}$ dem $Z$ weck multipliren wir unsere Gleichungen mit

$$
\frac{1}{4 \pi A} d \Omega \text {, }
$$

worin $d \Omega$ ein Volumenelement bedeutet, dann der Reihe nach mit $L, M, N, X, Y, Z$, addiren und integriren über einen durch eine geschlossene Fläche begrenzten Raum mit constantem $\varepsilon, \mu$.

Dann bekommen wir nach der Ausführung der partiellen Integration in dem ersten Integrale auf der rechten Seite:

$$
\begin{array}{r}
\frac{\partial}{\partial t} \iiint d \Omega\left\{\frac{\varepsilon}{8 \pi}\left(X^{2}+Y^{2}+Z^{2}\right)+\frac{\mu}{8 \pi}\left(L^{2}+M^{2}+N^{2}\right)\right\} \\
+\frac{1}{4 \pi} \iiint i \Omega \mu\left\{\frac{\partial \alpha}{\partial x}\left(M^{2}+N^{2}\right)+\frac{\partial \beta}{\partial y}\left(L^{2}+N^{2}\right)+\frac{\partial \gamma}{\partial z}\left(L^{2}+M^{2}\right)\right. \\
\left.-L M\left(\frac{\partial \alpha}{\partial y}+\frac{\partial \beta}{\partial x}\right)-M N\left(\frac{\partial \beta}{\partial z}+\frac{\partial \gamma}{\partial y}\right)-L N\left(\frac{\partial \gamma}{\partial x}+\frac{\partial \alpha}{\partial x}\right)\right\}, \\
+\frac{1}{4 \pi} \iiint d \Omega \varepsilon\left\{\frac{\partial \alpha}{\partial x}\left(Y^{2}+Z^{2}\right)+\frac{\partial \beta}{\partial y}\left(X^{2}+Z^{2}\right)+\frac{\partial \gamma}{\partial z}\left(X^{2}+Y^{2}\right)\right. \\
\left.-X Y\left(\frac{\partial \alpha}{\partial y}+\frac{\partial \beta}{\partial x}\right)-Y Z\left(\frac{\partial \beta}{\partial z}+\frac{\partial \gamma}{\partial y}\right)-X Z\left(\frac{\partial \gamma}{\partial x}+\frac{\partial \alpha}{\partial z}\right)\right\}, \\
+\frac{1}{4 \pi} \iiint d \Omega\left\{\frac{1}{2} \alpha \frac{\partial}{\partial x}\left[\mu\left(L^{2}+M^{2}+N^{2}\right)+\varepsilon\left(X^{2}+Y^{2}+Z^{2}\right)\right]\right. \\
+\frac{1}{2} \beta \frac{\partial}{\partial y}\left[\mu\left(L^{2}+M^{2}+N^{2}\right)+\varepsilon\left(X^{2}+Y^{2}+Z^{2}\right)\right] \\
\left.+\frac{1}{2} \gamma \frac{\partial}{\partial z}\left[\mu\left(L^{2}+M^{2}+N^{2}\right)+\varepsilon\left(X^{2}+Y^{2}+Z^{2}\right)\right]\right\},
\end{array}
$$




$$
\begin{aligned}
= & \frac{1}{4 \pi A} \iint d S\{\cos n x(M Z-N Y)+\cos n y(N X-L Z) \\
& +\cos n z(L Y-M X)\}, \\
& -\iiint d \Omega(u X+v Y+w Z),
\end{aligned}
$$

worin $d S$ ein Element der Oberfläche, $n$ die innere Normale bedeuten.

3. Beschäftigen wir uns nun mit der physikalischen Deutung der einzelnen Glieder dieser Gleichung. Offenbar bedeutet das erste Glied links die zeitliche Aenderung der gesamten Volumenenergie; das zweite und dritte Glied stellen offenbar die Arbeit der ponderomotorischen Kräfte dar, die als Drucke auf die Seiten eines elementaren Parallelepipedon $d \Omega$ wirksam sind.

Auf der rechten Seite stellt das erste Integral den Poynting'schen Ausdruck für die durch die Grenzfläche einströmende Energie dar, das zweite diejenige Energie, welche in Wärme nach dem Joule'schen Gesetz verwandelt wird. Es bleibt uns nur das Integral

$$
\begin{aligned}
\frac{1}{4 \pi} \iiint d \Omega & \left\{\frac{1}{2} \alpha \frac{\partial}{\partial x}\left[\mu\left(L^{2}+M^{2}+N^{2}\right)+\varepsilon\left(X^{2}+Y^{2}+Z^{2}\right)\right]\right. \\
+ & \frac{1}{2} \beta \frac{\partial}{\partial y}\left[\mu\left(L^{2}+M^{2}+N^{2}\right)+\varepsilon\left(X^{2}+Y^{2}+Z^{2}\right)\right] \\
& \left.+\frac{1}{2} \gamma \frac{\partial}{\partial z}\left[\mu\left(L^{2}+M^{2}+N^{2}\right)+\varepsilon\left(X^{2}+Y^{2}+Z^{2}\right)\right]\right\}
\end{aligned}
$$

übrig, von dessen Deutung auch der Gesamtwert der ponderomotorischen Kräfte abhängt.

4. Bemerken wir aber, dass, wie unsere Integrale zeigen, die uns interessirenden Kräfte von $\alpha, \beta, \gamma$ explicite unabhängig sind und dass also dieselben der Form nach unverändert bleiben sollen, auch wenn die Körperteile in Ruhe, ebenso wenn die elektromagnetischen Vorgänge statisch sind, so folgt unmittelbar, dass das genannte Integral nicht einfach als Arbeit der auf ein inneres Volumenelement wirkenden Kräfte zu deuten ist.

Wir müssen vielmehr das Integral durch partielle Integration in zwei Ausdrücke zerteilen, von denen nur der eine die in Betracht kommende Arbeit darstellt. 
In dieser Weise bekommt man

$$
\begin{gathered}
-\frac{1}{8 \pi} \iint d S\left\{\mu\left(L^{2}+M^{2}+N^{2}\right)+\varepsilon\left(X^{2}+Y^{2}+Z^{2}\right)\right\} \times \\
(\alpha \cos n x+\beta \cos n y+\gamma \cos n z), \\
-\frac{1}{8 \pi} \iiint d \Omega\left\{\mu\left(L^{2}+M^{2}+N^{2}\right)+\varepsilon\left(X^{2}+Y^{2}+Z^{2}\right)\right\} \times \\
\left(\frac{\partial \alpha}{\partial x}+\frac{\partial \beta}{\partial y}+\frac{\partial \gamma}{\partial z}\right) .
\end{gathered}
$$

Das erste Glied ist nun als diejenige Energieströmung zu betrachten, die von der Bewegung der Materie abhängt und die also eine Erweiterung des Poynting'schen Satzes darstellt ${ }^{1}$ ); was aber das Volumenintegral anbetrifft, so ist dasselbe mit den anderen die Arbeit darstellenden Integralen $\mathrm{zu}$ verbinden. Wir gelangen auf diese Weise zu dem zuerst von $\mathrm{Hertz}^{2}$ ) abgeleiteten Ausdruck der Arbeit von ponderomotorischen Kräften

$$
\begin{aligned}
& \iiint d \Omega\left\{X_{x} \frac{\partial \alpha}{\partial x}+Y_{y} \frac{\partial \beta}{\partial y}+Z_{z} \frac{\partial \gamma}{\partial z}\right. \\
&\left.\quad+X_{y}\left(\frac{\partial \alpha}{\partial y}+\frac{\partial \beta}{\partial x}\right)+Y_{z}\left(\frac{\partial \beta}{\partial z}+\frac{\partial \gamma}{\partial y}\right)+Z_{x}\left(\frac{\partial \gamma}{\partial x}+\frac{\partial \alpha}{\partial z}\right)\right\} \\
& X_{x}=X_{x}^{\prime}+X_{x}^{\prime \prime} \text { etc. } \quad \dot{X}_{y}=X_{y}^{\prime}+X_{y}^{\prime \prime} \text { etc. } \\
& X_{x}^{\prime}=\frac{\mu}{8 \pi}\left(-L^{2}+M^{2}+N^{2}\right), \quad X_{y}^{\prime}=Y_{x}^{\prime}=-\frac{\mu}{4 \pi} L M, \\
& Y_{y}^{\prime}=\frac{\mu}{8 \pi}\left(\quad L^{2}-M^{2}+N^{2}\right), \quad Y_{z}^{\prime}=Z_{y}^{\prime}=-\frac{\mu}{4 \pi} M N, \\
& Z_{z}^{\prime}=\frac{\mu}{8 \pi}\left(\quad L^{2}+M^{2}-N^{2}\right), \quad Z_{x}^{\prime}=X_{z}^{\prime}=-\frac{\mu}{4 \pi} N L, \\
& X_{x}^{\prime \prime}=\frac{\varepsilon}{8 \pi}\left(-X^{2}+Y^{2}+Z^{2}\right), \quad X_{y}^{\prime \prime}=Y_{x}^{\prime \prime}=-\frac{\varepsilon}{4 \pi} X Y, \\
& Y_{y}^{\prime \prime}=\frac{\varepsilon}{8 \pi}\left(\quad X^{2}-Y^{2}+Z^{2}\right), Y_{z}^{\prime \prime}=Z_{y}^{\prime \prime}=-\frac{\varepsilon}{4 \pi} Y Z, \\
& Z_{z}^{\prime \prime}=\frac{\varepsilon}{8 \pi}\left(\quad X^{2}+Y^{2}-Z^{2}\right), Z_{x}^{\prime \prime}=X_{z}^{\prime \prime}=-\frac{\varepsilon}{4 \pi} Z X,
\end{aligned}
$$

der nach der Ausführung der partiellen Integration die folgende Form annimmt:

1) Vgl. W. Wien, Wied. Ann. 47. p. 337. 1892.

2) H. Hertz, Wied. Ann. 41. p. 394. 1890. 


$$
\begin{aligned}
&-\iiint d \Omega\left\{\alpha\left(\frac{\partial X_{x}}{\partial x}+\frac{\partial Y_{x}}{\partial y}+\frac{\partial Z_{x}}{\partial x}\right)\right.+\beta\left(\frac{\partial X_{y}}{\partial x}+\frac{\partial Y_{y}}{\partial y}+\frac{\partial Z_{y}}{\partial z}\right) \\
&\left.+\gamma\left(\frac{\partial X_{z}}{\partial x}+\frac{\partial Y_{z}}{\partial y}+\frac{\partial Z_{z}}{\partial x}\right)\right\} \\
&-\iint d S\left\{\alpha\left(X_{x} \cos n x+X_{y} \cos n y+X_{z} \cos n z\right)\right. \\
&+\beta\left(Y_{x} \cos n x+Y_{y} \cos n y+Y_{z} \cos n z\right) \\
&\left.+\gamma\left(Z_{x} \cos n x+Z_{y} \cos n y+Z_{z} \cos n z\right)\right\} .
\end{aligned}
$$

5. Wir schliessen daraus, dass die zur Untersuchung stehenden ponderomotorischen Kräfte sich in zwei Kräftearten zerlegen lassen: mit den Componenten

$$
\begin{aligned}
& \equiv=-\frac{\partial X_{x}}{\partial x}-\frac{\partial Y_{x}}{\partial y}-\frac{\partial Z_{x}}{\partial z}, \\
& \mathrm{H}=-\frac{\partial X_{y}}{\partial x}-\frac{\partial Y_{y}}{\partial y}-\frac{\partial Z_{y}}{\partial z}, \\
& \mathrm{Z}=-\frac{\partial X_{x}}{\partial x}-\frac{\partial Y_{x}}{\partial y}-\frac{\partial Z_{x}}{\partial z}
\end{aligned}
$$

die auf innere Volumenelemente des Körpers wirkenden Kräfte, und mit den Componenten

$$
\begin{aligned}
& \bar{\Xi}_{n}=-\bar{X}_{x} \cos n x-\bar{X}_{y} \cos n y-\bar{X}_{z} \cos n z, \\
& \overline{\mathrm{H}}_{n}=-\bar{Y}_{x} \cos n x-\bar{Y}_{y} \cos n y-\bar{Y}_{z} \cos n z, \\
& \overline{\mathrm{Z}}_{n}=-\bar{Z}_{x} \cos n x-\bar{Z}_{y} \cos n y-\bar{Z}_{z} \cos n z
\end{aligned}
$$

die auf jeder Grenzfläche wirkenden, worin $\bar{X}_{x}, \ldots$ die Werte von $X_{x}, \ldots$ unendlich nahe an der Grenzfläche von der Seite der inneren Normale $n$ bedeuten.

6. Die Ausführung der Differentiation in den Ausdrücken von $\equiv, \ldots$ ergiebt nun

$$
\begin{aligned}
& \equiv=\frac{\mu}{4 \pi} L\left(\frac{\partial L}{\partial x}+\frac{\partial M}{\partial y}+\frac{\partial N}{\partial z}\right)+\frac{\varepsilon}{4 \pi} X\left(\frac{\partial X}{\partial x}+\frac{\partial Y}{\partial y}+\frac{\partial Z}{\partial z}\right) \\
& +\frac{\mu}{4 \pi} M\left(\frac{\partial L}{\partial y}-\frac{\partial M}{\partial x}\right)+\frac{\theta}{4 \pi} Y\left(\frac{\partial X}{\partial y}-\frac{\partial Y}{\partial x}\right) \\
& +\frac{\mu}{4 \pi} N\left(\frac{\partial L}{\partial z}-\frac{\partial N}{\partial x}\right)+\frac{\varepsilon}{4 \pi} Z\left(\frac{\partial X}{\partial z}-\frac{\partial Z}{\partial x}\right),
\end{aligned}
$$

analog für $H, Z$. 
Es sei unser Körper zunächst in Ruhe; wir lassen durch denselben sich eine elektromagnetische Störung fortpflanzen, die durch bekannte Gleichungen

$$
\begin{array}{lc}
A \mu \frac{\partial L}{\partial t}=\frac{\partial Z}{\partial y}-\frac{\partial Y}{\partial z}, & A \varepsilon \frac{\partial X}{\partial t}=\frac{\partial M}{\partial z}-\frac{\partial N}{\partial y}-4 \pi A u, \\
A \mu \frac{\partial M}{\partial t}=\frac{\partial X}{\partial z}-\frac{\partial Z}{\partial x}, & A \varepsilon \frac{\partial Y}{\partial t}=\frac{\partial N}{\partial x}-\frac{\partial L}{\partial z}-4 \pi A v, \\
A \mu \frac{\partial N}{\partial t}=\frac{\partial Y}{\partial x}-\frac{\partial X}{\partial y}, & A \varepsilon \frac{\partial Z}{\partial t}=\frac{\partial L}{\partial y}-\frac{\partial M}{\partial x}-4 \pi A w, \\
\frac{\partial L}{\partial x}+\frac{\partial M}{\partial y}+\frac{\partial N}{\partial z}=0, & \frac{\partial X}{\partial x}+\frac{\partial Y}{\partial y}+\frac{\partial Z}{\partial z}=0
\end{array}
$$

dargestellt wird. Dann folgen die Werte von $\equiv, H, Z$ in der Form

$$
\begin{aligned}
& \equiv=\frac{A \varepsilon \mu}{4 \pi} \frac{\partial}{\partial t}(M Z-N Y)+A \mu(M w-N v), \\
& \mathrm{H}=\frac{A \varepsilon \mu}{4 \pi} \frac{\partial}{\partial t}(N X-L Z)+A \mu(N u-L w), \\
& \mathrm{Z}=\frac{A \varepsilon \mu}{4 \pi} \frac{\partial}{\partial t}(I Y-M X)+A \mu(L v-M u),
\end{aligned}
$$

worin

$$
u=\frac{X}{k}, \quad v=\frac{Y}{k}, \quad w=\frac{Z}{k}
$$

ist und $k$ den specifischen Widerstand des Körpers bedeutet.

Um nun $\alpha, \beta, \gamma$ zu finden, sollen wir diese Kräfte als äussere Kräfte in die bekannten Differentialgleichungen der Bewegung elastischer Körper einführen; in gleicher Weise müssen $\bar{\Xi}_{n}, \overline{\mathrm{H}}_{n}, \overline{\mathrm{Z}}_{n}$ als äussere Kräfte in die Grenzbedingungen der elastischen Druckkräfte eingesetzt werden. Sind dabei $X, \ldots N$ periodisch veränderlich, so wird dasselbe auch für $\alpha, \beta, \gamma$ der Fall, sodass wir im allgemeinen elastische Schwingungen erhalten werden. Da aber, wie schon v. Helmholtz ${ }^{1}$ ) bemerkt hatte, $\equiv, \ldots Z_{n}$ Grössen zweiten Grades und bei regelmässigen Lichtschwingungen verschwindend kleine zweiter Ordnung sind, so werden auch $\alpha, \beta, \gamma$ ebenso klein und wir können daher den Einfluss von $\alpha, \beta, \gamma$ auf $L, M, N, X, Y, Z$ auch für die bestevacuirten Räume ausser acht lassen.2) Nur für den reinen Aether (sogar ohne Beharrungsvermögen) wäre

1) H. v. Helmholtz, Wied. Ann. 53. p. 139. 1894.

2) H. Hertz, Wied. Ann. 41. p. 398. 1890. 
die von diesen Kräften herrübrende Bewegung zu untersuchen, wie es v. Helmholtz gethan hat.

7. Nach diesen Ueberlegungen bieten für uns die momentanen Werte von $\Xi, \ldots \bar{\Xi}_{n}, \ldots$, also auch von $X_{x}, \ldots$ kein Interesse dar; wir werden daher unsere Aufgabe in der Weise beschränken können, dass wir immer nur die mittleren Werte der Kräfte für die ganze Schwingungsperiode in Rechnung ziehen.

Wir nehmen ferner an, dass $L, M, N, X, Y, Z$ die Form

$$
a \cos \left(b-\frac{2 \pi t}{T}\right)
$$

haben, worin $a, b$ Functionen der Coordinaten sind, von der Zeit aber unabhängig; dann reducirt sich die Berechnung der Mittelwerte von $\equiv, \ldots$ auf die Integration der Ausdrücke von der Form

$$
\begin{aligned}
\frac{1}{T} \int_{t}^{t+T} A \frac{\partial}{\partial t}\{\cos (b & \left.\left.-\frac{2 \pi t}{T}\right) \cos \left(b^{\prime}-\frac{2 \pi t}{T}\right)\right\} d t \\
& +\frac{1}{T} \int_{t}^{t+T} B \cos \left(b-\frac{2 \pi t}{T}\right) \cos \left(b^{\prime}-\frac{2 \pi t}{T}\right) d t
\end{aligned}
$$

was offenbar giebt

$$
\frac{B}{2} \cos \left(b-b^{\prime}\right) \text {. }
$$

Es können also bei den gewöhnlichen Lichtschwingungen die Mittelwerte von 三, ... nur dann verschwinden, wenn

$$
\frac{M Z-N Y}{k}=0, \quad \frac{N X-L Z}{k}=0, \quad \frac{L Y-M X}{k}=0,
$$

also wenn entweder

$$
M Z-N Y=0, \quad N X-L Z=0, \quad L Y-M X=0,
$$

oder

$$
k=\infty \text {. }
$$

Da ferner $M Z-N Y, \ldots$ die Componenten des bekannten Poynting'schen Vectors darstellen, so sehen wir daraus, das auch in den Leitern die Werte von $\equiv, \ldots$ innig mit der Bewegung der Energie verbunden sind. ${ }^{1}$ )

1) Vgl. H. v. Helmholtz, Wied. Ann. 53. p. 139. 1894. 
Von jetzt ab wollen wir im weiteren unter $X_{x}, \ldots \equiv, \ldots$ $\equiv_{n}, \ldots X^{2} \ldots$ immer die in der angegebenen Weise berechneten Mittelwerte verstehen.

8. Wir stellen uns nun $z$ wei sich an der Ebene $z=0$ angrenzende Medien vor und wählen die $y z$-Ebene für die Einfallsebene einer linearpolarisirten ebenen Lichtwelle, sodass wir setzen

$$
Y=Z=0, \quad L=0 .
$$

Von der Seite der negativen $z$ liege ein nichtleitendes Medium, für welches offenbar gilt

$$
\begin{gathered}
\equiv=0, \quad \mathrm{H}=0, \quad \mathrm{Z}=0, \\
X_{x}=\frac{\mu}{8 \pi}\left(\quad M^{2}+N^{2}\right)-\frac{\varepsilon}{8 \pi} X^{2}, \quad X_{y}=0, \\
Y_{y}=\frac{\mu}{8 \pi}\left(-M^{2}+N^{2}\right)+\frac{\varepsilon}{8 \pi} X^{2}, \quad Y_{z}=-\frac{\mu}{4 \pi} M N, \\
Z_{z}=\frac{\mu}{8 \pi}\left(M^{2}-N^{2}\right)+\frac{\varepsilon}{8 \pi} X^{2}, \quad Z_{x}=0,
\end{gathered}
$$

und an der Ebene $z=0$, wo $\cos n z=-1$ ist, haben wir

$$
\bar{\Xi}_{n}=0, \quad \overline{\mathrm{H}}_{n}=+\bar{Y}_{z}, \quad \overline{\mathrm{Z}}_{n}=+\bar{Z}_{z} .
$$

Setzen wir in leicht verständlichen Bezeichnungen

$$
\begin{aligned}
X_{0} & =\mathfrak{A}_{0} \cos 2 \pi\left(\frac{z \cos \varphi+y \sin \varphi}{\lambda}-\frac{t}{T}\right), \\
X_{r} & =\mathfrak{A}_{r} \cos \left[2 \pi\left(\frac{-z \cos \varphi+y \sin \varphi}{\lambda}-\frac{t}{T}\right)+\delta\right]
\end{aligned}
$$

und rechnen wir $\mathfrak{A}_{r}$ immer positiv, so folgt für die Mittelwerte $X^{2}=\left(X_{0}+X_{r}\right)^{2}$ etc. auf der Ebene $z=0$

$$
\begin{aligned}
\frac{\mu}{8 \pi} \Pi^{2} & =\frac{1}{2} \frac{\varepsilon}{8 \pi}\left(\mathfrak{I}_{0}^{2}+\mathfrak{M}_{r}^{2}+2 \mathfrak{A}_{0} \mathfrak{A}_{r} \cos \delta\right), \\
\frac{\mu}{8 \pi} \bar{M}^{2} & =\frac{1}{2} \frac{\varepsilon}{8 \pi}\left(\mathfrak{H}_{0}^{2}+\mathfrak{A}_{r}^{2}-2 \mathfrak{A}_{0} \mathfrak{A}_{r} \cos \delta\right) \cos ^{2} \varphi, \\
\frac{\mu}{8 \pi} N^{2} & =\frac{1}{2} \frac{\varepsilon}{8 \pi}\left(\mathfrak{I}_{0}^{2}+\mathfrak{A}_{r}^{2}+2 \mathfrak{A}_{0} \mathfrak{A}_{r} \cos \delta\right) \sin ^{2} \varphi, \\
\frac{\mu}{4 \pi} M N & =-\frac{\varepsilon}{8 \pi}\left(\mathfrak{A}_{0}^{2}-\mathfrak{A}_{r}^{2}\right) \sin \varphi \cos \varphi .
\end{aligned}
$$


Bezeichnen wir ferner durch $e_{0}$, $e_{r}$ die Energie in der Volumeneinheit der einfallenden bez. der reflectirten Strahlen, so ist bekanntlich

$$
\frac{\varepsilon}{8 \pi} \mathfrak{A}_{0}^{2}=e_{0}, \quad-\frac{\varepsilon}{8 \pi} \mathfrak{A}_{r}^{2}=e_{r},
$$

und wir bekommen

$$
\begin{array}{ll}
\bar{X}_{x}=-2 \sqrt{e_{0} e_{r}} \cos \delta \cos ^{2} \varphi, & \bar{X}_{y}=0, \\
\bar{Y}_{y}=2 \sqrt{e_{0} e_{r}} \cos \delta+\left(e_{0}+e_{r}\right) \sin ^{2} \varphi, & \overline{Y_{z}}=\left(e_{0}-e_{r}\right) \sin \varphi \cos \varphi, \\
\bar{Z}_{z}=\quad\left(e_{0}+e_{r}\right) \cos ^{2} \varphi, & \overline{Z_{x}}=0
\end{array}
$$

und folglich

$$
\Xi_{n}=0, \quad \overline{\mathrm{H}}_{n}=\left(e_{0}-e_{r}\right) \sin \varphi \cos \varphi, \quad \bar{Z}_{n}=\left(e_{0}+e_{r}\right) \cos ^{2} \varphi .
$$

9. Die Reflexionsebene erleidet also einen von dem auffallenden und reflectirten Lichte herrührenden schiefen Druck $p$ in der Einfallsebene, dessen Richtung mit der $z$-Axe einen Winkel $\psi$ bildet, sodass

$$
\begin{gathered}
\operatorname{tg} \psi=\frac{e_{0}-e_{r}}{e_{0}+e_{r}} \operatorname{tg} \varphi, \\
p=\cos \varphi \sqrt{e_{0}^{2}+e_{r}^{2}+2 e_{0} e_{r} \cos 2 \varphi .}
\end{gathered}
$$

Führen wir das Reflexionsvermögen $J_{\varphi}$ der Ebene $z=0$ ein, so folgt $e_{r}=J_{p} e_{0}$, und

$$
\begin{gathered}
\operatorname{tg} \psi=\frac{1-J_{\varphi}}{1+J_{\varphi}} \operatorname{tg} \varphi, \\
p=e_{0} \cos \varphi \sqrt{1+J_{\varphi}^{2}+2 J_{\varphi} \cos 2 \varphi} .
\end{gathered}
$$

Für den Fall vollkommener Reflexion haben wir $J_{\varphi}=1$ und

$$
\begin{aligned}
& \psi=0, \\
& \left.p=2 e_{0} \cos ^{2} \varphi,^{1}\right)
\end{aligned}
$$

für vollkommene Absorption wird $J_{\varphi}=0$ und

$$
\begin{aligned}
& \psi=\varphi, \\
& p=e_{0} \cos \varphi,
\end{aligned}
$$

endlich für normale Incidenz ist

$$
\begin{aligned}
& \psi=0, \\
& p=e_{0}\left(1+J_{0}\right) .
\end{aligned}
$$

1) Vgl. die Betrachtungen von Boltzmann, l. c. p. 292. 
Daraus folgt gerade das Resultat von Maxwell für $J_{0}=0$ und von Boltzmann für $J_{0}=1$.

10. Wir stellen uns vor, dass die einfallenden Strahlen einen Kreiscylinder bilden, dessen Axe die $z=0$-Ebene in dem Coordinatenanfang schneidet; diese Strahlen beleuchten auf der Ebene $z=0$ eine Ellipse, deren Axen in der Richtung von $y$ und $x$-Axe fallen. Die reflectirten Strahlen bilden offenbar einen ähnlichen Cylinder. Ausserhalb der Schnittpunkte der beiden Cylinder haben wir, wie leicht zu ersehen ist, für die einfallenden Strahlen:

$$
\begin{array}{ll}
X_{x}=0, & X_{y}=0, \\
Y_{y}=e_{0} \sin ^{2} \varphi, & Y_{z}=e_{0} \sin \varphi \cos \varphi, \\
Z_{z}=e_{0} \cos ^{2} \varphi, & Z_{x}=0 .
\end{array}
$$

Eine einfache Coordinatentransformation zeigt, dass diese Kräfte nur eine Resultirende geben, und zwar einen mit der Wellennormale zusammenfallenden Druck, dessen Wert $e_{0}$ beträgt, wie es schon Maxwell auf anderem Wege gefunden hat. Ein solcher Druck kann keine auf der Mantelfläche des Cylinders wirkende Kraft ergeben; dasselbe Resultat gilt natürlich auch für die reflectirten Strahlen. Es bleiben also im ersten Medium nur Kräfte in den Schnittpunkten der beiden cylindrischen Mantelfächen.

Ihre grösste Wirkung erhalten diese Kräfte bei der normalen Incidenz. Dann sind die Mittelwerte

$$
\begin{aligned}
& \frac{\varepsilon}{8 \pi} X^{2}=\frac{1}{2} \frac{\varepsilon}{8 \pi}\left(\mathfrak{U}_{0}^{2}+\mathfrak{A}_{r}^{2}+: 2 \mathfrak{A}_{0} \mathfrak{A}_{r} \cos \left(\frac{4 \pi z}{\lambda}-\delta\right)\right), \\
& \frac{\mu}{8 \pi} M^{2}=\frac{1}{2} \frac{\varepsilon}{8 \pi}\left(\mathfrak{U}_{0}^{2}+\mathfrak{A}_{r}^{2}-2 \mathfrak{A}_{0} \mathfrak{A}_{r} \cos \left(\frac{\mathbf{4} \pi}{\lambda}-\delta\right)\right)
\end{aligned}
$$

und folglich

$$
\begin{array}{ll}
X_{x}=-2 \sqrt{e_{0} e_{r}} \cos \left(\frac{4 \pi z}{\lambda}-\delta\right), & X_{y}=0, \\
Y_{y}=2 \sqrt{e_{0} e_{r}} \cos \left(\frac{4 \pi z}{\lambda}-\delta\right), & Y_{z}=0, \\
Z_{z}=e_{0}+e_{r}, & Z_{x}=0
\end{array}
$$

und auf der Mantelfläche des Cylinders

$$
\Xi_{n}=-X_{x} \cos n x, \quad \mathrm{H}_{n}=-Y_{y} \cos n y, \quad \bar{Z}_{n}=0 \text {. }
$$


11. Bisher haben wir die Wellenbewegung an der Seite der positiven $z$ ganz ausser acht gelassen. Jetzt wenden wir uns zu den hier wirkenden ponderomotorischen Kräften. Das Medium mit $z>0$ sehen wir als eine Platte an, deren Dicke $h$ und deren Breite so gross sind, dass kein in die Platte gebrochener Strahl aus derselben heraustritt.

Ist nun unsere Platte unbeweglich, so wird dieselbe durch die im Inneren und auf der vorderen Basis wirkende Kräfte deformirt, was seinerseits die elastische Reaction des Platten. materiales hervorrufen wird. Diese elastischen Kräfte zu berechnen ist cine sehr schwierige Aufgabe. Es ist aber auch von vornherein klar, dass diese elastische Reaction nicht ohne Einfluss auf die Resultirende der an der Ebene $z=0$ wirkenden Kräfte bleiben kann. Auch im Falle der normalen Incidenz verschwinden die Kräfte $X_{x}, Y_{y}$ im Inneren der Platte nicht.

Es wird aber unsere Aufgabe sich sehr vereinfachen, wenn wir uns die Platte als vollkommen beweglich vorstellen; natürlich nehmen wir dann das die Platte umgebende Medium flüssig an. Dann haben wir nur die resultirende Kraft bez. Kräftepaare zu berechnen, die die Platte als ganzes zu verschieben bez. drehen streben. Nun aber sind die Kräfte $\equiv, H, Z$ im Inneren der Platte und $\bar{\Xi}_{n}, \overline{\mathrm{H}}_{n}, \bar{Z}_{n}$ gerade derart, dass

$$
\iint \bar{\Xi}_{n} d S+\iiint \equiv d \Omega=0
$$

und analog für $\mathrm{H}, \mathrm{Z}$

$$
\iint\left(y \equiv_{n}-x \overline{\mathrm{H}}_{n}\right) d S+\iiint(y \equiv-x \mathrm{H}) d \Omega=0,
$$

und analog für die anderen Drehungsmomente. In diesem Falle haben wir also nur mit der Wellenbewegung im ersten Medium zu thun und die von dem Lichte herrührenden ponderomotorischen Kräfte setzen sich aus einem "Lichtdruck“ zusammen, den die Platte erleidet, und noch aus gewissen Kräften, die im ersten Medium wirksam sind und die Strömungen im flüssigen Dielektricum hervorrufen werden.

12. Wir wollen noch den Fall einer vollkommen durchsichtigen Platte untersuchen, und zwar soll für $z>h$ wieder dasselbe Medium sein, wie für $z<0$. 
Der Einfachheit wegen nehmen wir jetzt $\mathscr{\varphi}=0$, also betrachten den Fall der normalen Incidenz, und suchen die die vollkommen bewegliche Platte in Bewegung $\mathrm{zu}$ setzen strebenden Kräfte. Da dieselben nach dem früheren nur von dem Lichte ausser der Platte abhängen, so haben wir mit der Lichtbewegung in der Platte nichts zu thun. Es sei $e_{1}$ die mittlere Energie der $\nabla$ olumeneinheit der Strahlen, die im ersten Medium sich in der Richtung von der Platte fortpflanzen; ebenso sei $e_{d}$ die Energie der Strahlen, die aus der Platte bei $z=k$ herausgetreten sind. Dann haben wir

$$
\begin{array}{lll}
z=0, & \bar{Z}_{0}=e_{0}+e_{1}, & \cos n z=-1, \\
z=h, & \bar{Z}_{l}=-e_{d}, & \cos n z=+1
\end{array}
$$

und die die Platte in der Richtung der $z$-Axe bewegende Kraft, der Lichtdruck, wird

Bekanntlich ist

$$
p=e_{0}+e_{1}-e_{d} .
$$

woraus folgt

$$
\begin{aligned}
& e_{d}=e_{0} \frac{1-J_{0}}{1+J_{0}} \\
& e_{0}=e_{1}+e_{d},
\end{aligned}
$$

oder

$$
p=2 e_{0}\left(1-\frac{1-J_{0}}{1+J_{0}}\right)
$$

$$
p=e_{0} \frac{4 J_{0}}{1+J_{0}} .
$$

Bei $J=\frac{1}{3}$ würde folgen $p=e_{0}$, gerade wie in dem Falle einer absolut schwarzen Fläche. Da ferner

$$
J_{0}=\left(\frac{N-1}{N+1}\right)^{2}
$$

ist, so können wir auch schreiben

$$
p=2 e_{0} \frac{(N-1)^{2}}{N^{2}+1} .
$$

Für das Glas in der Luft ist $N=1,5$; für den Diamanten ist $N=2,5$; das giebt im ersten Falle

in dem zweiten

$$
p=0,15 \cdot e_{0}
$$

$$
p=0,62 . e_{0} .
$$


Für eine lichtabsorbirende Platte haben wir abgeleitet

$$
p=e_{0}\left(1+J_{0}\right)
$$

für blankes Platin beträgt $J_{0}$ etwa 0,70 , daraus folgt

$$
p=1,7 . e_{0} .
$$

Wir betrachten dabei die Platte offenbar so dick, dass die Erscheinungen der „dünnen Blättchen“ ausser acht gelassen werden können.

13. Für den zweiten Hauptfall der Polarisation müssen wir

$$
M=0, \quad N=0, \quad X=0
$$

setzen, dann ist:

$$
\begin{array}{ll}
X_{x}=\frac{\varepsilon}{8 \pi}\left(Y^{2}+Z^{2}\right)-\frac{\mu}{8 \pi} L^{2}, & X_{y}=0, \\
Y_{y}=\frac{\varepsilon}{8 \pi}\left(-Y^{2}+Z^{2}\right)+\frac{\mu}{8 \pi} L^{2}, & Y_{z}=-\frac{\varepsilon}{4 \pi} Y Z, \\
Z_{z}=\frac{\varepsilon}{8 \pi}\left(Y^{2}-Z^{2}\right)+\frac{\mu}{8 \pi} L^{2}, & Z_{x}=0,
\end{array}
$$

und bekommen wir die früheren Werte von $X_{x}, \ldots$ nur mit dem Unterschiede, dass $e$ einen anderen numerischen Wert darstellt. Daraus ist zu schliessen, dass alle unsere Resultate auch jetzt bestehen bleiben. Dann aber ist auch ein directer Uebergang zu dem Falle des natürlichen Lichtes erlaubt, nur muss man unter $e_{0}, e_{r}, J_{p}$ die entsprechenden Grössen für natürliches Licht verstehen.

14. Ein ganz besonderes Interesse bietet der Fall dar, wenn das auffallende Licht senkrecht zur Einfallsebene polarisirt unter dem Polarisationswinkel $\bar{\varphi}$ auffällt. Dann bekommen wir keine Reflexion, sodass

$$
e_{d}=e_{0}
$$

wird und wir haben folgende Componente der die Platte zu bewegen bez. zu drehen strebenden Kräfte:

$$
\begin{aligned}
& z=0, \quad \bar{\Xi}_{0}=0, \quad \overline{\mathrm{H}}_{0}=e_{0} \sin \bar{\varphi} \cos \bar{\varphi}, \quad \bar{Z}_{0}=e_{0} \cos ^{2} \bar{\varphi}, \\
& z=h, \quad \bar{\Xi}_{h}=0, \quad \overline{\mathrm{H}}_{h}=-e_{0} \sin \phi \cos \bar{\varphi}, \quad \bar{Z}_{h}=-e_{0} \cos ^{2} \bar{\varphi} \text {. }
\end{aligned}
$$

Die Resultirende dieser Kräfte ist offenbar Null; es ergiebt sich nur ein Drehungsmoment, dessen Componenten wir 
$M_{x}, M_{y}, M_{z}$ bezeichnen wollen. Es seien ferner $\bar{x}_{0}, \bar{y}_{0}, \bar{z}_{0}$ und $\bar{x}_{h}, \bar{y}_{h}, \bar{z}_{h}$ die Coordinaten der entsprechenden Punkte der Ebenen $z=0$ bez. $z=h, d S_{0}, d S_{h}$ die Flächenelemente; dann ist

$$
M_{x}=\iint\left(\bar{y}_{0} \bar{Z}_{0}-\bar{z}_{0} \overline{\mathrm{H}}\right) d S_{0}+\iint\left(\bar{y}_{h} \bar{Z}_{h}-\bar{z}_{h} \overline{\mathbf{H}}_{h}\right) d S_{h},
$$

analoge Ausdrücke für $M_{y}, M_{z}$; nun haben wir offenbar

$$
\bar{x}_{h}=\bar{x}_{0}, \quad \bar{y}_{h}=\bar{y}_{0}+h \operatorname{tg} \bar{\varphi}_{1}, \quad \bar{z}_{h}=\bar{z}_{0}+h, \quad d S_{0}=d S_{h},
$$

woraus folgt

$$
\begin{aligned}
& M_{x}=\iint\left[\bar{y}_{0}\left(\bar{Z}_{0}+\bar{Z}_{h}\right)-\bar{z}_{0}\left(\mathrm{H}_{0}+\overline{\mathrm{H}}_{h}\right)\right] d S_{0}+h \iint\left(\bar{Z}_{h} \operatorname{tg} \bar{\varphi}_{1}\right.\left.-\overline{\mathrm{H}}_{h}\right) d S_{0}, \\
& M_{y}=\iint\left[\bar{x}_{0}\left(\overline{\mathrm{H}}_{0}+\overline{\mathrm{H}}_{h}\right)-\bar{y}_{0}\left(\bar{\Xi}_{0}+\bar{\Xi}_{h}\right)\right] d S_{0}-h \operatorname{tg}{\overline{\varphi_{1}}}_{1} \iiint l S_{0}, \\
& M_{z}=\iint\left[\bar{z}_{0}\left(\bar{\Xi}_{0}+\bar{\Xi}_{h}\right)-\bar{x}_{0}\left(\overline{\mathbf{Z}}_{0}+\mathrm{Z}_{h}\right)\right] d S_{0}+h \bar{\Xi}_{h} \iint d S_{0},
\end{aligned}
$$

oder

$$
M_{x}=h S_{0}\left(\bar{Z}_{h} \operatorname{tg} \bar{\varphi}_{1}-\overline{\mathrm{H}}_{h}\right), \quad M_{y}=0, \quad M_{z}=0,
$$

worin $S_{0}$ die beleuchtete Fläche auf der Ebene $z=0, \bar{\varphi}_{1}$ den Brechungswinkel bedeuten.

Die Substitution der Werthe von $\bar{Z}_{h}, \bar{H}_{h}$ ergiebt ferner

oder da

$$
M_{x}=e_{0} S_{0} h \cos \bar{\varphi}\left(\sin \bar{\varphi}-\cos \bar{\varphi} \operatorname{tg} \tilde{\varphi}_{1}\right),
$$

ist, so folgt endlich

$$
\operatorname{tg} \bar{\varphi}=N=\operatorname{ctg} \bar{\varphi}_{1}
$$

$$
M_{x}=e_{0} S_{0} h \frac{N^{2}-1}{N\left(N^{2}+1\right)} .
$$

Für Glas in der Luft berechnet man daraus $M_{x}=0,26 e_{0} S_{0} h$; für den Diamanten $M_{x}=0,29 e_{0} S_{0} h$. Das gewonnene Drehungsmoment ist offenbar eine ganz neue Folgerung der elektromagnetischen Lichttheorie.

15. Wir wollen nun den Fall der sphärischen Wellen untersuchen. Es liege der Coordinatenanfang in dem leuchtenden Punkte, die Reflexionsebene sei die Ebene $z=h$; wir behalten 
frühere Richtungen der Coordinatenaxen und nehmen die auffallende Kugelwelle elliptisch polarisirt. Setzen wir ferner

$$
\begin{gathered}
r^{2}=x^{2}+y^{2}+z^{2}, \\
\theta_{n}=2 \pi\left(\frac{r}{\lambda}-\frac{t}{T}\right)+\delta_{n}, \quad n=1,2,3,
\end{gathered}
$$

so ist zu nehmen für die auffallende Welle ${ }^{1}$ ) (mit gewöhnlicher Annäherung)

$$
\begin{aligned}
& X_{0}=\frac{1}{r}\left(\mathfrak{B} \frac{\partial r}{\partial z} \cos \theta_{2}-\mathfrak{E} \frac{\partial r}{\partial y} \cos \theta_{3}\right), \\
& Y_{0}=\frac{1}{r}\left(\mathfrak{C} \frac{\partial r}{\partial x} \cos \theta_{3}-\mathfrak{A} \frac{\partial r}{\partial z} \cos \theta_{1}\right), \\
& Z_{0}=\frac{1}{r}\left(\mathfrak{A} \frac{\partial r}{\partial y} \cos \theta_{1}-\mathfrak{B} \frac{\partial r}{\partial x} \cos \theta_{2}\right),
\end{aligned}
$$

woraus folgt

$$
\begin{aligned}
& L_{0}=\frac{1}{r}\left(\alpha_{1} \cos \theta_{1}+\alpha_{2} \cos \theta_{2}+\alpha_{3} \cos \theta_{3}\right), \\
& M_{0}=\frac{1}{r}\left(\beta_{1} \cos \theta_{1}+\beta_{2} \cos \theta_{2}+\beta_{3} \cos \theta_{3}\right), \\
& N_{0}=\frac{1}{r} \cdot\left(\gamma_{1} \cos \theta_{1}+\gamma_{2} \cos \theta_{2}+\alpha_{3} \cos \theta_{3}\right),
\end{aligned}
$$

dabei ist

$$
\begin{aligned}
& \alpha_{1}=-\sqrt{\frac{8}{\mu}} \mathfrak{A}\left\{\left(\frac{\partial r}{\partial y}\right)^{2}+\left(\frac{\partial r}{\partial x}\right)^{2}\right\}, \quad \alpha_{2}=\sqrt{\frac{\varepsilon}{\mu}} \mathfrak{B} \frac{\partial r}{\partial y} \frac{\partial r}{\partial x}, \\
& c_{3}=\sqrt{\frac{\varepsilon}{\mu}} \mathfrak{C} \frac{\partial r}{\partial z} \frac{\partial r}{\partial x}, \\
& \beta_{1}=\sqrt{\frac{\delta}{\mu}} \mathfrak{A} \frac{\partial r}{\partial x} \frac{\partial r}{\partial y}, \quad \beta_{2}=-\sqrt{\frac{\delta}{\mu}} \mathfrak{B}\left\{\left(\frac{\partial r}{\partial z}\right)^{2}+\left(\frac{\partial r}{\partial x}\right)^{2}\right\}, \\
& \beta_{3}=\sqrt{\frac{\varepsilon}{\mu} @ \frac{\partial r}{\partial z} \frac{\partial r}{\partial y}}, \\
& \gamma_{1}=\sqrt{\frac{\varepsilon}{\mu}} \mathfrak{A} \frac{\partial r}{\partial x} \frac{\partial r}{\partial z}, \quad \gamma_{2}=\sqrt{\frac{\varepsilon}{\mu}} \mathfrak{B} \frac{\partial r}{\partial y} \frac{\partial r}{\partial z}, \\
& \gamma_{3}=-\sqrt{\frac{\varepsilon}{\mu}}\left(\left\{\left\{\frac{\partial r}{\partial x}\right)^{2}+\left(\frac{\partial r}{\partial y}\right)^{2}\right\} .\right.
\end{aligned}
$$

1) P. Volkmann, Vorlesungen über die Theorie des Lichtes p. 65. Leipzig 1891. 
Für die reflectirte Kugelwelle müssen wir nehmen

$$
r^{2}=x^{2}+y^{2}+(2 h-z)^{2},
$$

und $\mathfrak{A}, \mathfrak{B}, \mathfrak{C}, \theta_{1}, \theta_{2}, \theta_{3}$ bez. durch $\mathfrak{A}_{r}, \mathfrak{V}_{r}, \mathfrak{夭}_{r}, \theta_{1}^{\prime}, \theta_{2}{ }^{\prime}, \theta_{3}{ }^{\prime}$ ersetzen; es ist dabei offenbar für $z=h$

$$
\frac{\partial r^{\prime}}{\partial x}=\frac{\partial r}{\partial x}, \quad \frac{\partial r^{\prime}}{\partial y}=\frac{\bar{\partial} r}{\partial y}, \quad \frac{\partial r^{\prime}}{\partial z}=-\frac{\partial r}{\partial z} ; \quad \bar{r}=\bar{r}^{\prime}
$$

und die Berechnung von den Mittelwerten $\bar{X}^{2}=\left(\bar{X}_{0}+\bar{X}_{r}\right)^{2}, \ldots$, wenn auch langweilig, bietet doch keine Schwierigkeit dar.

In dieser Weise berechnen wir die Normalcomponente des Druckes auf die Ebene $z=h \mathrm{zu}$

$$
\bar{Z}_{h}=\left(\bar{e}_{0}+\bar{e}_{r}\right)\left(\frac{\partial r}{\partial \tilde{r}}\right)^{2}=\left(\bar{e}_{0}+\bar{e}_{r}\right) \cos ^{2} \varphi
$$

worin $\bar{e}_{0}, \bar{e}_{r}$ die mittlere Energie der Volumeneinheit der einfallenden bez. der reflectirten Strahlen bei der Ebene $z=h$ bedeuten; dabei sind natürlich diese Grössen, wie auch $\varphi$, für verschiedene Strahlen verschieden.

16. Es sei nun $i_{0}$ diejenige Energie, die die auffallende Welle in einer Secunde auf die Einheit der Fläche $z=h$ bringt; die analoge Grösse für die reflectirte Welle sei $i_{r}$; bezeichnen wir mit $V$ die Lichtgeschwindigkeit, so folgt, wie leicht zu ersehen ist,

$$
\begin{gathered}
\bar{e}_{0}=\frac{i_{0}}{V \cos \varphi}, \quad \bar{e}_{r}=\frac{i_{r}}{V \cos \varphi}, \\
Z_{h}=\frac{i_{0}+i_{r}}{V} \cos \varphi .
\end{gathered}
$$

Diese Beziehung bleibt auch dann gelten, wenn man eine strahlende Fläche hat; dann ist nur $i_{0}+i_{r}$ durch die ausgestrahlte Energie zu ersetzen.

Betrachten wir z. B. eine absolut schwarze Fläche, deren $1 \mathrm{qcm}$ in der Zeiteinheit normal die Energie $i$ ausstrahlt, dann ist für den Winkel, der zwischen $\varphi$ und $\varphi+d \varphi$ liegt,

$$
i_{\varphi}=i \cos \varphi
$$

und die entsprechende Druckcomponente wird

$$
Z_{h}=\frac{i_{\varphi} \cos \varphi}{V}=\frac{i \cos ^{2} \varphi}{V} .
$$


Die Menge solcher Strahlen ist bekanntlich $2 \pi \sin \varphi d \varphi$, und folglich beträgt der Druck, zum Flächenelement $d S$ normal,

$$
p_{\lambda}=\frac{2 \pi}{V} i \int_{0}^{\pi / 2} \cos ^{2} \varphi \sin \varphi d \varphi, \quad p_{\lambda}=\frac{2}{3} \frac{\pi i}{V} .
$$

Stellt nun $E_{\lambda}$ das Gesetz der Energieverteilung im Spectrum des absolut schwarzen Körpers dar, so haben wir

$$
\begin{gathered}
i=E_{\lambda} d \lambda, \\
p_{\lambda}=\frac{2 \pi}{3} \frac{E_{\lambda}}{V} d \lambda .
\end{gathered}
$$

Der Gesamtdruck ergiebt sich daraus zu

$$
p=\frac{2 \pi}{3} \int_{0}^{\infty} \frac{E_{\lambda}}{V} d \lambda .
$$

Ist $V$ von $\lambda$ unabhängig, wie es für den Aether der Fall ist, so folgt einfach

$$
V=\frac{1}{A}, \quad p=\frac{2 \pi}{3} E A, \quad E=\int_{0}^{\infty} E_{\lambda} d \lambda .
$$

Kasan, December 1900.

(Eingegangen 31. Januar 1901.) 